\title{
The Emerging Role of PARP Inhibitors in the Treatment of Epithelial Ovarian Cancer
}

\author{
Lilian T. Gien and Helen J. Mackay \\ Princess Margaret Hospital, University of Toronto, Toronto, ON, Canada M5G 2M9 \\ Correspondence should be addressed to Helen J. Mackay, helen.mackay@uhn.on.ca \\ Received 21 July 2009; Accepted 22 October 2009 \\ Academic Editor: Charles F. Levenback
}

Copyright ( $) 2010$ L. T. Gien and H. J. Mackay. This is an open access article distributed under the Creative Commons Attribution License, which permits unrestricted use, distribution, and reproduction in any medium, provided the original work is properly cited.

Poly(ADP-ribose) polymerase-1 (PARP-1) is an important novel target in cancer therapy. This enzyme is essential in the repair of single-stranded breaks in DNA via the base excision repair pathway. Drugs which inhibit PARP are emerging as a promising new class of anticancer agents particularly effective against tumors which have lost homologous recombination (HR) through loss of functional BRCA1 and BRCA2. PARP inhibitors potentially represent a major breakthrough for patients with hereditary BRCA-associated cancers. Furthermore their role in sporadic epithelial ovarian cancer is emerging with identification of additional subpopulations of women who may benefit a priority. This paper will summarize the mechanism of action of PARP inhibition and its role in the treatment of BRCA1- and 2-associated cancers. We will then expand on the broader relevance and future directions for PARP inhibition in the clinical setting.

\section{Introduction}

Epithelial ovarian cancer (EOC) is the fifth leading cause of death in women in North America [1]. Despite the efficacy of platinum-based chemotherapy, over $75 \%$ of women with stage III/IV EOC ultimately relapse and die from their disease. Median survival for women whose disease does not respond or in whom duration of response is short is less than 12 months [2]. Traditional cytotoxics topotecan and liposomal doxorubicin demonstrate only modest efficacy in women with platinum resistant EOC and are associated with significant toxicity [3]. New therapeutic approaches, and the ability to identify patients groups who will derive benefit from them, are urgently required.

Over recent years the investigation of DNA repair in cancer cells has been a very active area of translational research. All cells have a number of overlapping pathways to protect the genome from DNA damage which occurs as a result of normal cell cycling, environmental insults, or cytotoxic chemotherapy. It is well recognized that when mutations occur within these DNA repair pathways there is an increased risk of malignant transformation and chemotherapy resistance [4]. Much research has focused on protecting cells from DNA damage and/or restoring DNA repair function. However, emerging data suggest that the concept of "synthetic lethality," that is, exploiting the vulnerability of cancer cells which have lost one mechanism of DNA repair by targeting a second pathway, may be a particularly attractive therapeutic approach. Poly(ADPribose) polymerase (PARP) is an enzyme which plays an important role in the recognition and repair of single-strand DNA breaks via the base excision repair (BER) pathway [5]. Over the last few years it has become apparent that in cells which have lost BRCA1 or BRCA2, components of a second DNA repair pathway, homologous recombination (HR), are particularly sensitive to PARP inhibition. These data suggest that PARP inhibitors may be particularly useful for the treatment of women with hereditary BRCA1/2-associated EOC $[6,7]$. Targeted therapy using PARP inhibitors has become an important novel strategy for treating those with hereditary ovarian cancer. Furthermore the identification of other subpopulations of women with EOC who may benefit from this approach is an active area of research. 
This paper will outline the mechanism of PARP inhibition and discuss this in relation to loss of BRCA function. We will summarize the preclinical and clinical evidence from the most recent studies and discuss future directions for PARP inhibition in EOC.

\section{BRCA1 and BRCA2}

BRCA1 or BRCA2 mutations occur in $0.1-0.8 \%$ of the general population and are inherited in an autosomal dominant manner [8]. They are well recognized to have a higher incidence in certain ethnic groups, such as women of Ashkenazi Jewish descent [9]. Women carrying a mutation in BRCA1 have a lifetime risk of developing ovarian cancer of between 40 and 50\%, while those carrying a BRCA2 mutation have a slightly lower risk of 10-20\% [10]. Over the past ten years, the focus of management for those identified as BRCA1/2 mutation carriers has been on cancer prevention and early cancer detection. However, despite prophylactic measures to reduce risk of EOC, many BRCA1/2 carriers will already have cancer at the time their mutation is diagnosed.

The BRCA1 gene is located on chromosome 17q21, while BRCA2 is located on chromosome $13 q 12[11,12]$. BRCA1 and BRCA2 play major roles in the repair of DNA doublestrand breaks (DSBs) by homologous recombination (HR). $\mathrm{HR}$ repairs DSBs that occur in late $\mathrm{S}$ and G2 phase of the cell cycle and also has a key role in repairing DSBs that result from unrepaired single-strand break (SSB) [13]. BRCA1 signals the presence of DSBs, while BRCA2 is directly involved in the mechanism of HR. In the absence of BRCA1 or BRCA2, alternative DNA repair pathways are used, which result in chromosomal instability and cell death. Normal cells of carriers are usually heterozygote with loss of the second allele occurring during tumorigenesis in the tumor cells of these women [14].

Currently, the treatment of patients with BRCAassociated EOC is identical to those with sporadic EOC. However, even prior to the emergence of the PARP inhibitors, data suggested that cancers associated with BRCA mutations responded differently to chemotherapy [15]. Tan et al. compared 22 BRCA-positive patients with EOC to 44 nonhereditary EOC controls in a matched case-control study. They found that BRCA-positive patients have higher response rates to first line platinum-based treatment $(81.8 \%$ versus $43.2 \%, P=.004)$, subsequent lines of platinum-based treatments (second line, $91.7 \%$ versus $40.9 \%, P=.004$ ), longer tumor-free intervals between relapses, and improved overall survival $(8.4$ versus 2.9 years, $P<.002)[16]$. This data implies that different strategies may be required in this group of women.

\section{Poly(ADP-Ribose) Polymerase Inhibitors}

There are currently 17 members of the PARP superfamily identified [17]. PARP-1 is the most studied enzyme, which is involved in the repair of SSBs of DNA by the base excision repair (BER) pathway [5]. Targeting the nuclear enzyme PARP-1 represents a new and novel approach to the treatment of EOC and appears to be particularly promising for those carrying mutations in the BRCA1 and 2 genes [14].

Cells utilize several overlapping DNA repair mechanisms to maintain the integrity of the genome. PARP-1 activation occurs in response to metabolic, chemical, or radiationinduced DNA SSBs and forms part of the BER pathway $[18,19]$. PARP-1 detects and signals the presence of an SSB by binding to DNA adjacent to the damage. Once bound, PARP- 1 catalyzes the cleavage of the coenzyme nicotinamide adenine dinucleotide (NAD+) into nicotinamide and ADPribose to produce highly negatively charged branched chains of poly(ADP-ribose) (PAR). A multiprotein repair complex is then formed including repair enzymes DNA ligase III, the DNA polymerase pol B, and scaffolding proteins such as XRCC1 (X-ray repair cross-complementing 1). Following ADP-ribosylation, PARP-1 has reduced affinity for DNA and is released. After repair, the PAR polymers are degraded via poly(ADP-ribose) glycohydrolase (PARG) [14] (Figure 1). The role of PARP-1 may not be limited to just SSB repair; roles in the DSB repair response have also been proposed [20].

The new generation of PARP inhibitors inhibits PARP by competitive inhibition of NAD+. In the preclinical setting, PARP-1 inhibitors enhance the cytotoxic effects of ionizing radiation and cytotoxic chemotherapy [21]. Additionally, in the preclinical setting, the use of PARP-1 inhibitors as single agents did not cause any measurable toxicity, but the combination of PARP-1 inhibitor with temozolomide in the tumorbearing mice caused significant toxicity [22]. There did not seem to be a correlation, however, between the antitumor activity and the toxicity of the PARP inhibitor-temozolomide combinations, suggesting that toxicity and chemosensitization were by different mechanisms. While promising in combination with other agents, PARP inhibitors appear to be particularly potent in patients who have defects in DNA repair.

In a normal cell, PARP-1 inhibition leads to failure of SSB repair, resulting in the formation of a DSB in the DNA when a replication fork encounters the SSB. Thus the DSB can be repaired by HR and the fidelity of the genome maintained. However, in cells carrying defects in BRCA1/2, HR is defective, resulting in an attempted repair of the DSB by the more error prone nonhomologous end joining (NHEJ) pathway [18]. As a result, the cell acquires lethal levels of damage and cellular viability is lost, a prime example of "synthetic lethality" with the malignant cell able to function with the loss of one DNA repair mechanism (HR) but ceasing to be viable with the loss of a second (BER) $[23,24]$. As most BRCA1/2 carriers have one normal allele, the hope was that inhibition of PARP would be selective for tumor cells.

In 2005, two preclinical papers demonstrated the sensitivity of BRCA1- and BRCA2-deficient cell lines to PARP inhibition [6, 7]. The first paper by Bryant et al. demonstrated reduced survival of BRCA2-deficient cell lines with four PARP inhibitors. They concluded that BRCA2deficient cells were sensitive to PARP inhibition, and that monotherapy with one of these agents could selectively kill cancer cells [6]. In the same year, Farmer et al. 


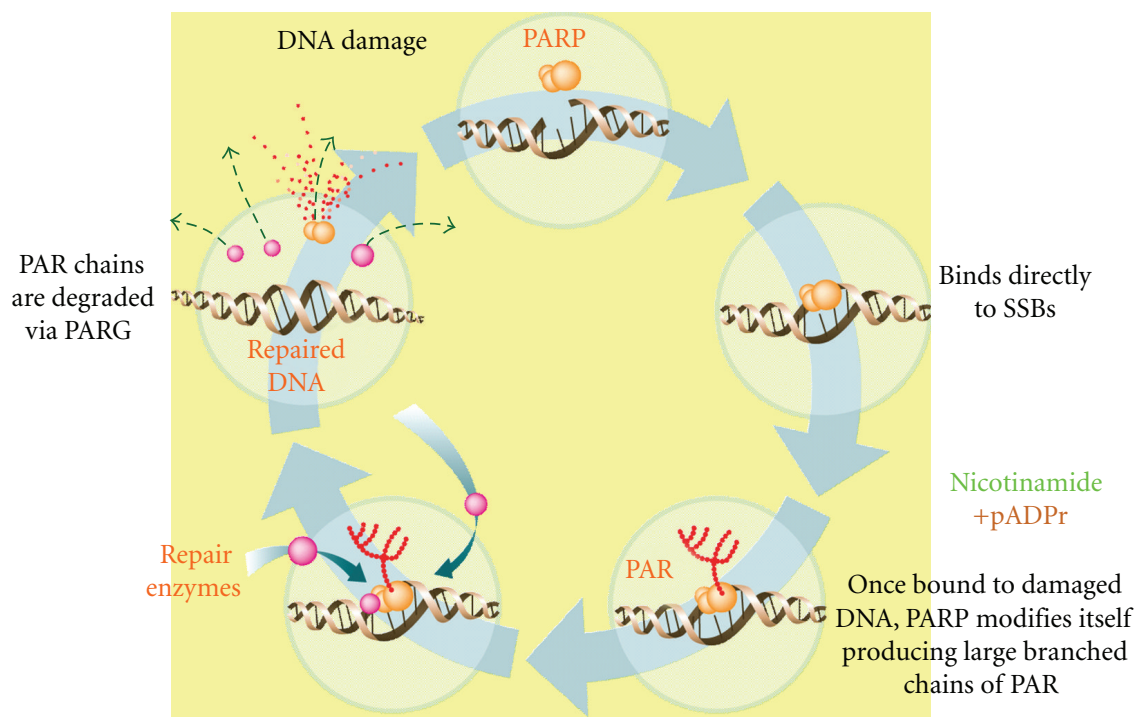

Figure 1: The role of PARP in the repair of single-strand DNA breaks via the base excision repair pathway.

demonstrated how both BRCA1- and BRCA2-deficient cells lines were sensitive to inhibition of PARP-1, and that BRCA2 deficient cells were more than 1000 times more sensitive to nanomolar concentrations of PARP inhibitor [7]. Both of these papers demonstrated how homozygotes (tumor cells) are sensitive to the mechanism of PARP inhibition; whereas heterozygotes (the rest of the patient's cells) are insensitive to this mechanism and should not exhibit toxicity. These findings from two independent groups using different chemical classes of PARP inhibitors on different BRCAdeficient cell lines were the first to suggest the potent effect of PARP inhibition.

\section{Clinical Evidence in Phase I and II Trials}

A number of PARP inhibitors have entered the clinic in both intravenous and oral formulations. The four which are furthest along in terms of development are AGO14699 (Pfizer), AZD2281 (AstraZeneca), ABT-888 (Abbott), and BSI-201 (BI Par), and all four of these compounds demonstrate profound inhibition of PARP-1.

Olaparib (AZD2281, KU- 0059436, AstraZeneca) is an oral small-molecule PARP inhibitor. The first clinical evidence demonstrating the sensitivity of BRCA-mutated cancers to PARP inhibitor monotherapy was presented in a study by Yap et al. in 2007 [25]. This phase I trial included 44 patients, of which 11 patients had a BRCA mutation associated cancer. Dose escalation was guided by toxicity, pharmacokinetic and pharmacodynamic data. Based on the encouraging antitumor activity, many in whom had BRCA1/2 mutations, the trial was subsequently expanded to concentrate on cancers in patients with BRCA mutations and was presented in 2008 by Fong et al. [26], followed by publication of the manuscript in 2009 [27]. The drug was well tolerated in both BRCA mutated and normal populations. Most toxicities were grade 1-2 ( $\geq 95 \%)$, consisting of fatigue (28\%), nausea (28\%), vomiting (18\%), loss of taste (13\%), and anorexia (12\%). Grade 3-4 toxicities were rare, consisting of myelosuppression $(\leq 5 \%)$, nausea and vomiting (2-3\%), and dizziness or mood changes (2$3 \%)$ [27]. Of the 60 patients that were enrolled and treated, 19 of 23 BRCA-positive carrriers were evaluable. 12 of the $19(63 \%)$ had a clinical benefit from olaparib, with radiologic or tumor marker responses, or stable disease for 4 months or more [27]. Patient response was seen in those receiving a minimum of $100 \mathrm{mg}$ twice daily up to $400 \mathrm{mg}$ twice daily. Response was the greatest in patients with platinum-sensitive disease, although duration of response was the same regardless of the platinum-free interval [26].

Recently data was presented from a phase II study of olaparib in women with advanced EOC with known mutations in BRCA1/2 [28]. Two patient cohorts received continuous oral olaparib in 28-day cycles; 33 patients received $400 \mathrm{mg}$ orally twice daily, while 24 patients received $100 \mathrm{mg}$ twice daily. The choice of dosing and schedule was based on the phase I trial above [25]. The objective response rate measured by RECIST criteria was 33\% at the $400 \mathrm{mg}$ dose, and $12.5 \%$ at the $100 \mathrm{mg}$ dose, suggesting that there may be a dose response effect. The toxicity profile was mainly mild, consisting of grade 1 or 2 nausea $(44 \%)$ and fatigue $(35 \%)$, with few grade 3 or 4 toxicities. Interestingly, although numbers were low, in this study there appeared to be a higher response rate in platinum resistant patients (38\% versus 14\%), which was opposite to that observed in the earlier phase I study (Table 1), where response was the greatest in platinum-sensitive patients. Laboratory studies have previously suggested that platinum resistant patients may reacquire BRCA function [29] thus potentially making them resistant to the effects of PARP inhibition. Taken together, the clinical data suggest that we still have a lot to learn with regard to target populations and the role of PARP inhibition. Furthermore, data from 
TABLE 1: Responses rates of women with epithelial ovarian cancer to olaparib (AZD2281) by platinum sensitivity in Phase I (Fong et al.) [26] and Phase II trials (Audeh et al.) [28].

\begin{tabular}{lcccccc}
\hline & \multicolumn{2}{c}{ No. evaluable } & \multicolumn{2}{c}{ Responders by RECIST (\%) } & \multicolumn{2}{c}{ Responders by RECIST or GCIG (\%) } \\
& Phase I [26] & Phase II [28] & Phase I [26] & Phase II [28] & Phase I [26] & Phase II [28] \\
\hline Total & 46 & 33 & $13(28 \%)$ & $11(33 \%)$ & $21(46 \%)$ & $20(61 \%)$ \\
Platinum sensitive (>6 months) & 10 & 7 & $5(50 \%)$ & $1(14 \%)$ & $8(80 \%)$ & - \\
Platinum resistant ( $\leq 6$ months) & 25 & 26 & $8(32 \%)$ & $10(38 \%)$ & $11(44 \%)$ & - \\
Platinum refractory & 11 & - & $0(0 \%)$ & - & $2(18 \%)$ & - \\
\hline
\end{tabular}

the phase II study appears to give an early indication that response (both RECIST and CA125) may be greater in those patients with BRCA2 mutations. This would be in line with the known mechanism of action of the two BRCA proteins as BRCA2 plays a key role in the repair pathway; whereas BRCA1 functions as a signaling molecule [30]. This phase II study concluded that oral olaparib is well tolerated and highly active in advanced, chemotherapyrefractory BRCA-deficient EOC, with greater activity seen at a higher dose of $400 \mathrm{mg}$ twice daily. The optimal patient group with respect to platinum sensitivity has not been defined.

Reassuringly in the clinical studies there does not appear to be an increase in toxicity between BRCA mutation carriers compared to noncarriers, supporting the theory that PARP inhibitors should not result in increased toxicity to heterozygote cells $[6,7]$.

These recent phase I and phase II trials are particularly promising for patients with BRCA-associated EOC. Further phase II trials are currently underway which will help further elucidate the role and potential for this new targeted therapy.

\section{PARP Inhibitors in Sporadic Ovarian Cancers}

BRCA-associated EOC is associated with only $10 \%$ of all ovarian cancers. However, loss of BRCA1/2 function is not exclusive to inheriting a mutation in the BRCA1/2 genes [31]. The results seen in known BRCA1 and 2 mutation carriers may also be relevant to the sporadic EOC patient population.

Epigenetic gene inactivation is a well-recognized phenomenon with $31 \%$ of EOC exhibiting aberrant methylation of the BRCA1 promoter [32]. Furthermore, genetic or epigenetic events occurring in other components of the HR pathway can be found in sporadic EOC [15, 33]. These tumors seem to be similar to BRCA1- or BRCA2-mutated tumors, even though they do not have mutations to either of these genes, a concept called "BRCAness." [15, 33]. One molecular characterization study suggested that over $50 \%$ of patients with high-grade EOC had loss of BRCA function, either by genetic or epigenetic events [34]. Studies have shown that the loss of functional proteins in the HR pathway may lead these cells to be sensitive to PARP inhibition [35]. Identification of "BRCA-like" EOC populations who may benefit from this new therapy through the identification and validation of biomarkers is an active area of ongoing research.

\section{Future Directions}

At least 6 PARP inhibitors, including AG0146999 (Pfizer) and MK4827 (Merck), are under investigation either as single agents and/or in combination with other agents or treatment modalities. Phase II studies in women with advanced EOC in both BRCA1/2 mutation carriers and high-grade EOC of unknown BRCA status are ongoing, many incorporating translational research questions which are vital to our understanding of the biology of PARP inhibition. Currently, olaparib is being evaluated in a randomized phase II trial comparing this agent with pegylated liposomal doxorubicin in patients with BRCA-mutated EOC with a platinum-free interval of 0-12 months [36].

Early data combining PARP inhibitors with cytotoxics suggested that the combinations may be toxic and that substantial dose reductions of the cytotoxic agents may be required [37]. Intriguingly, a randomized study in women with triple negative breast cancer presented at this year's American Society of Clinical Oncology (ASCO) suggests that this may not always be the case. Patients were randomized to receive either gemcitabine $1000 \mathrm{mg} / \mathrm{m}^{2}$ and carboplatin AUC 2 on days 1 and 8 with or without the PARP inhibitor BSI-201 [38]. In this study there was no difference in the rates of toxicity or dose adjustments between the two arms. Response rates were significantly higher $(P=$ .002) for women receiving the PARP inhibitor. Currently many combination studies are underway; the results are awaited with interest. Combination studies in women with both hereditary and sporadic EOC are expected in the future.

Further defining the role of PARP inhibitors in the clinic is ongoing. Olaparib is being evaluated in a randomized placebo-controlled trial as a maintenance therapy in patients with sporadic EOC at high risk of early recurrence [39]. Furthermore, some suggest that PARP inhibitors could be used to prevent cancers in patients who are BRCA mutation carriers [40]. This approach, however, requires careful consideration and some caution with the potential for the development of drug resistance in long-term use of PARP inhibitors.

Investigation of the PARP inhibitors in the nonhereditary EOC population is very active with both the impact of treatment on patients without BRCA defects and the search for populations of women who have lost functional proteins in the HR pathway. Investigation of PARP inhibitor resistance and ways to overcome this resistance are emerging fields. 


\section{Conclusions}

We are living in exciting times as our knowledge of tumor cell biology expands and new agents become available. As we move into the era of personalized medicine, the emerging data regarding the use of PARP inhibitors in patients with BRCA-associated EOC are encouraging and inspiring. Expansion and identification of further patient groups who will benefit from this approach are a priority. Over the next few years we expect to see an explosion in the publication of studies exploring the use and role of PARP inhibitors in the clinic. Careful clinical trial design, and the development and validation of biomarkers are essential if we are to make the optimal use of these exciting agents and improve outcome for women with EOC.

\section{References}

[1] S. A. Cannistra, "Cancer of the ovary," The New England Journal of Medicine, vol. 351, no. 24, pp. 2519-2529, 2004.

[2] S. A. Cannistra, "Is there a "best" choice of second-line agent in the treatment of recurrent, potentially platinum-sensitive ovarian cancer?" Journal of Clinical Oncology, vol. 20, no. 5, pp. 1158-1160, 2002.

[3] A. N. Gordon, J. T. Fleagle, D. Guthrie, D. E. Parkin, M. E. Gore, and A. J. Lacave, "Recurrent epithelial ovarian carcinoma: a randomized phase III study of pegylated liposomal doxorubicin versus topotecan," Journal of Clinical Oncology, vol. 19, no. 14, pp. 3312-3322, 2001.

[4] P. Peltomaki, "Role of DNA mismatch repair defects in the pathogenesis of human cancer," Journal of Clinical Oncology, vol. 21, no. 6, pp. 1174-1179, 2003.

[5] V. Schreiber, F. Dantzer, J.-C. Ame, and G. de Murcia, "Poly(ADP-ribose): novel functions for an old molecule," Nature Reviews Molecular Cell Biology, vol. 7, no. 7, pp. 517$528,2006$.

[6] H. E. Bryant, N. Schultz, H. D. Thomas, et al., "Specific killing of BRCA2-deficient tumours with inhibitors of poly(ADPribose) polymerase," Nature, vol. 434, no. 7035, pp. 913-917, 2005.

[7] H. Farmer, H. McCabe, C. J. Lord, et al., "Targeting the DNA repair defect in BRCA mutant cells as a therapeutic strategy," Nature, vol. 434, no. 7035, pp. 917-921, 2005.

[8] H. A. Risch, J. R. McLaughlin, D. E. C. Cole, et al., "Population BRCA1 and BRCA2 mutation frequencies and cancer penetrances: a kin-cohort study in Ontario, Canada," Journal of the National Cancer Institute, vol. 98, no. 23, pp. 1694-1706, 2006.

[9] E. Levy-Lahad, R. Catane, S. Eisenberg, et al., "Founder BRCA1 and BRCA2 mutations in Ashkenazi Jews in Israel: frequency and differential penetrance in ovarian cancer and in breast-ovarian cancer families," American Journal of Human Genetics, vol. 60, no. 5, pp. 1059-1067, 1997.

[10] D. Ford, D. F. Easton, D. T. Bishop, S. A. Narod, and D. E. Goldgar, "Risks of cancer in BRCA1-mutation carriers," The Lancet, vol. 343, no. 8899, pp. 692-695, 1994.

[11] K. Gudmundsdottir and A. Ashworth, "The roles of BRCA1 and BRCA2 and associated proteins in the maintenance of genomic stability," Oncogene, vol. 25, no. 43, pp. 5864-5874, 2006.

[12] G. Stewart and S. J. Elledge, "The two faces of BRCA2, a FANCtastic discovery," Molecular Cell, vol. 10, no. 1, pp. 2-4, 2002.
[13] Q. Zhong, C.-F. Chen, S. Li, et al., "Association of BRCA1 with the hRad50-hMre11-p95 complex and the DNA damage response," Science, vol. 285, no. 5428, pp. 747-750, 1999.

[14] Y. Drew and H. Calvert, "The potential of PARP inhibitors in genetic breast and ovarian cancers," Annals of the New York Academy of Sciences, vol. 1138, pp. 136-145, 2008.

[15] A. N. J. Tutt, C. J. Lord, N. McCabe, et al., "Exploiting the DNA repair defect in BRCA mutant cells in the design of new therapeutic strategies for cancer," Cold Spring Harbor Symposia on Quantitative Biology, vol. 70, pp. 139-148, 2005.

[16] D. S. P. Tan, C. Rothermundt, K. Thomas, et al., "“BRCAness" syndrome in ovarian cancer: a case-control study describing the clinical features and outcome of patients with epithelial ovarian cancer associated with BRCA1 and BRCA2 mutations," Journal of Clinical Oncology, vol. 26, no. 34, pp. 55305536, 2008.

[17] J.-C. Ame, C. Spenlehauer, and G. de Murcia, "The PARP superfamily," BioEssays, vol. 26, no. 8, pp. 882-893, 2004.

[18] J. H. J. Hoeijmakers, "Genome maintenance mechanisms for preventing cancer," Nature, vol. 411, no. 6835, pp. 366-374, 2001.

[19] D. D'Amours, S. Desnoyers, I. D'Silva, and G. G. Poirier, "Poly(ADP-ribosyl)ation reactions in the regulation of nuclear functions," Biochemical Journal, vol. 342, no. 2, pp. 249-268, 1999.

[20] N. Schultz, E. Lopez, N. Saleh-Gohari, and T. Helleday, "Poly(ADP-ribose) polymerase (PARP-1) has a controlling role in homologous recombination," Nucleic Acids Research, vol. 31, no. 17, pp. 4959-4964, 2003.

[21] R. C. Calabrese, R. Almassy, S. Barton, et al., "Anticancer chemosensitization and radiosensitization by the novel poly(ADP-ribose) polymerase-1 inhibitor AG14361," Journal of the National Cancer Institute, vol. 96, no. 1, pp. 56-67, 2004.

[22] H. D. Thomas, C. R. Calabrese, M. A. Batey, et al., "Preclinical selection of a novel poly(ADP-ribose) polymerase inhibitor for clinical trial," Molecular Cancer Therapeutics, vol. 6, no. 3, pp. 945-956, 2007.

[23] W. G. Kaelin Jr., "The concept of synthetic lethality in the context of anticancer therapy," Nature Reviews Cancer, vol. 5, no. 9, pp. 689-698, 2005.

[24] A. Ashworth, "A synthetic lethal therapeutic approach: poly(ADP) ribose polymerase inhibitors for the treatment of cancers deficient in DNA double-strand break repair," Journal of Clinical Oncology, vol. 26, no. 22, pp. 3785-3790, 2008.

[25] T. A. Yap, D. S. Boss, P. C. Fong, et al., "First in human phase 1 pharmacokinetic and pharmacodynamic study of KU$0059436(\mathrm{Ku})$, a small molecule inhibitor of poly(ADP-ribose) polymerase (PARP) in cancer patients including BRCA1/2 mutation carriers," Journal of Clinical Oncology, vol. 25, supplement, no. 18, 2007.

[26] P. C. Fong, D. Boss, C. Carden, et al., "AZD2281 (KU0059436), a PARP (poly ADP-ribose polymerase) inhibitor with single agent anticancer activity in patients with BRCA deficient ovarian cancer: results from a phase I study," Journal of Clinical Oncology, vol. 26, supplement, p. 295S, 2008.

[27] P. C. Fong, D. S. Boss, T. A. Yap, et al., "Inhibition of poly(ADP-ribose) polymerase in tumors from BRCA mutation carriers," The New England Journal of Medicine, vol. 361, no. 2, pp. 123-134, 2009.

[28] M. W. Audeh, R. T. Penson, M. Friedlander, et al., "Phase II trial of the oral PARP inhibitor olaparib (AZD2281) in BRCA-deficient advanced ovarian cancer," Journal of Clinical Oncology, vol. 27, supplement, p. 15S, 2009. 
[29] S. L. Edwards, R. Brough, C. J. Lord, et al., "Resistance to therapy caused by intragenic deletion in BRCA2," Nature, vol. 451, no. 7182, pp. 1111-1115, 2008.

[30] A. Tutt and A. Ashworth, "The relationship between the roles of BRCA genes in DNA repair and cancer predisposition," Trends in Molecular Medicine, vol. 8, no. 12, pp. 571-576, 2002.

[31] L. Hughes-Davies, D. Huntsman, M. Ruas, et al., "EMSY links the BRCA2 pathway to sporadic breast and ovarian cancer," Cell, vol. 115, no. 5, pp. 523-535, 2003.

[32] R. L. Baldwin, E. Nemeth, H. Tran, et al., "BRCA1 promoter region hypermethylation in ovarian carcinoma: a populationbased study," Cancer Research, vol. 60, no. 19, pp. 5329-5333, 2000.

[33] N. Turner, A. Tutt, and A. Ashworth, "Hallmarks of "BRCAness" in sporadic cancers," Nature Reviews Cancer, vol. 4, no. 10, pp. 814-819, 2004.

[34] J. Z. Press, A. De Luca, N. Boyd, et al., "Ovarian carcinomas with genetic and epigenetic BRCA1 loss have distinct molecular abnormalities," BMC Cancer, vol. 8, article 17, 2008.

[35] N. McCabe, N. C. Turner, C. J. Lord, et al., "Deficiency in the repair of DNA damage by homologous recombination and sensitivity to poly(ADP-ribose) polymerase inhibition," Cancer Research, vol. 66, no. 16, pp. 8109-8115, 2006.

[36] NCT00628251, http://clinicaltrials.gov/.

[37] R. Plummer, C. Jones, M. Middleton, et al., "Phase I study of the poly(ADP-ribose) polymerase inhibitor, AG014699, in combination with temozolomide in patients with advanced solid tumors," Clinical Cancer Research, vol. 14, no. 23, pp. 7917-7923, 2008.

[38] J. O’Shaughnessy, J. Osborne, M. Pippen, et al., "Efficacy of BSI-201, a poly(ADP-ribose) polymerase-1 (PARP1) inhibitor, in combination with gemcitabine/carboplatin (G/C) in patients with metastatic triple-negative breast cancer (TNBC): results of a randomized phase II trial," Journal of Clinical Oncology, vol. 27, supplement, no. 18, 2009.

[39] NCT00753545, http://clinicaltrials.gov/.

[40] T. Hay, H. Jenkins, O. J. Sansom, N. M. B. Martin, G. C. M. Smith, and A. R. Clarke, "Efficient deletion of normal BRCA2-deficient intestinal epithelium by poly(ADP-ribose) polymerase inhibition models potential prophylactic therapy," Cancer Research, vol. 65, no. 22, pp. 10145-10148, 2005. 


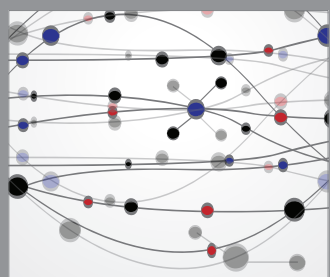

The Scientific World Journal
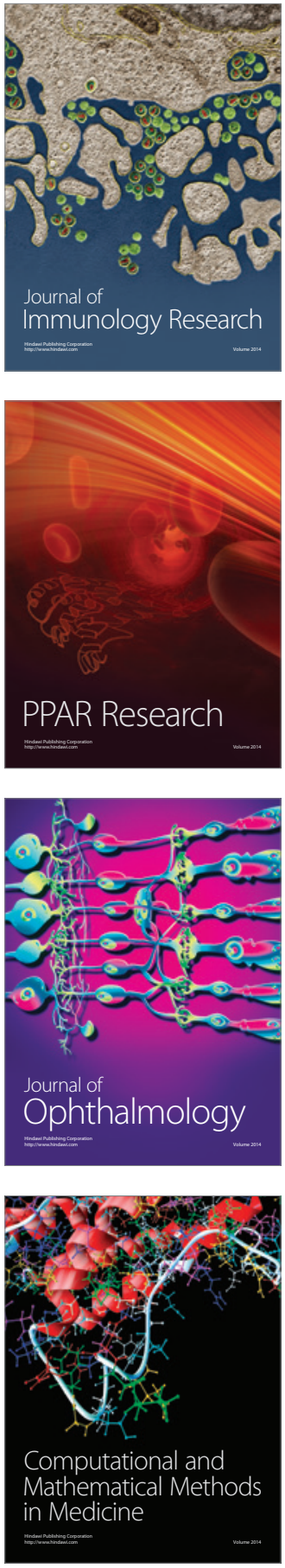

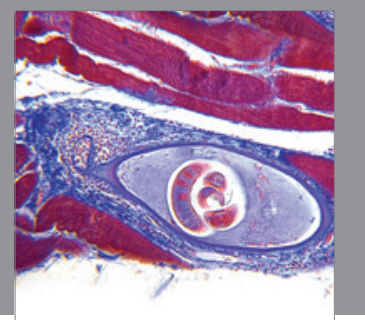

Gastroenterology

Research and Practice
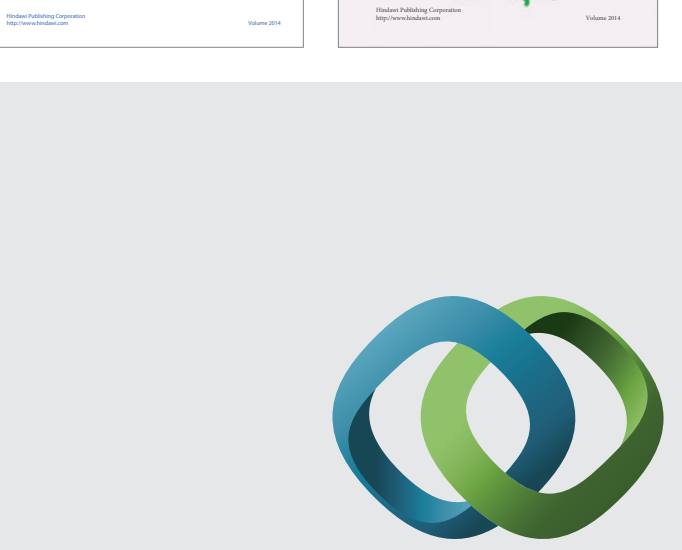

\section{Hindawi}

Submit your manuscripts at

http://www.hindawi.com
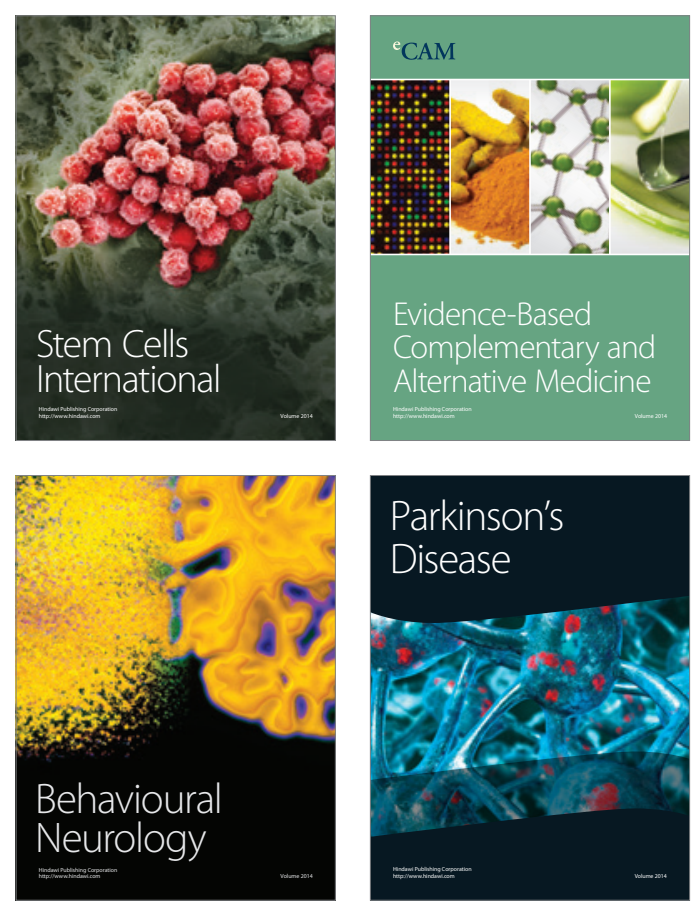

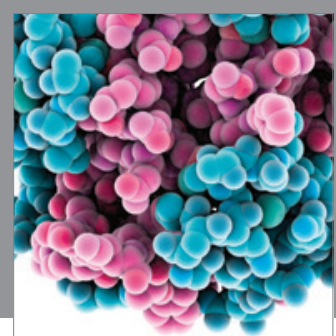

Journal of
Diabetes Research

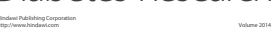

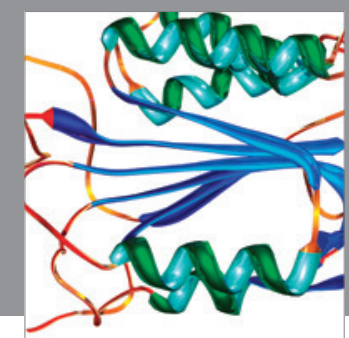

Disease Markers
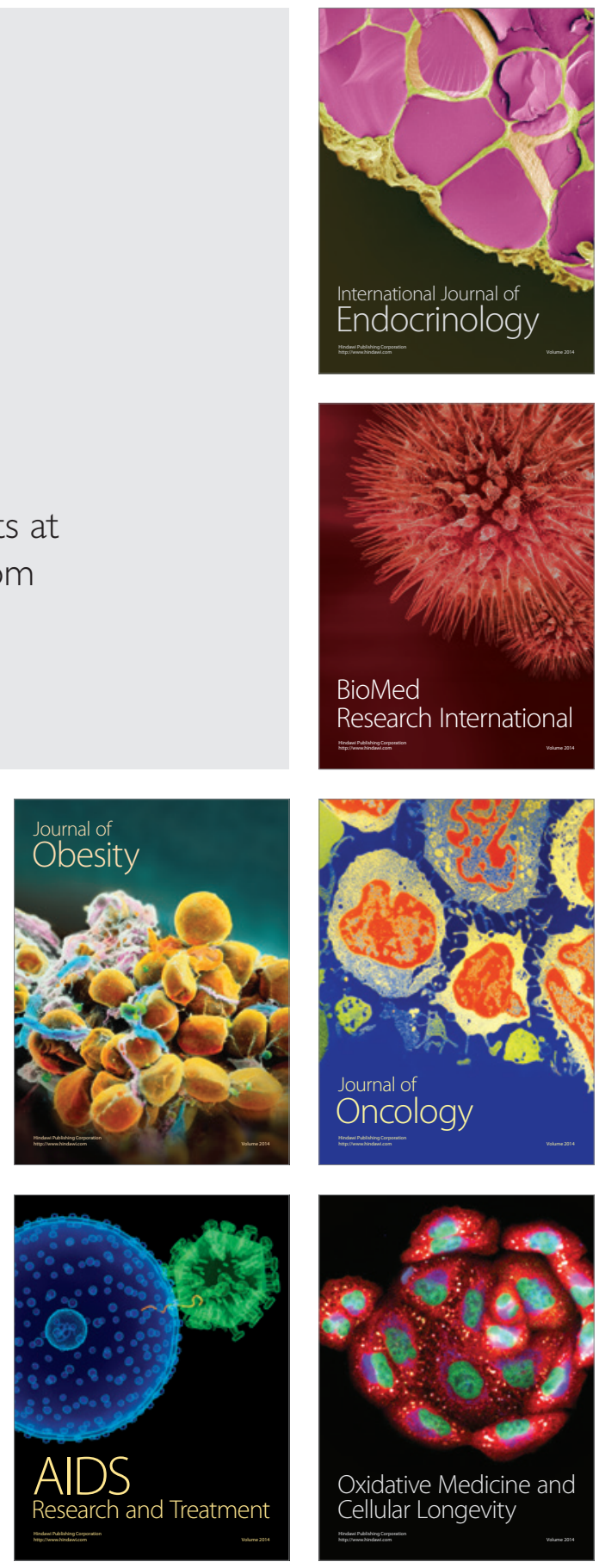